\title{
Altered hinge conformations in APP transmembrane helix mutants may affect enzyme-substrate interactions of $\boldsymbol{y}$-secretase
}

\author{
Mara Silber ${ }^{1}$, Manuel Hitzenberger ${ }^{2}$, Martin Zacharias² ${ }^{2}$ Claudia Muhle-Goll*1,3
}

\footnotetext{
${ }^{1}$ Karlsruhe Institute of Technology, Institute for Biological Interfaces 4, P.O. Box 3640, 76021 Karlsruhe, Germany. ${ }^{2}$ Physics Department T38, Technical University of Munich, James-Frank-Str. 1, 85748 Garching, Germany ${ }^{3}$ Karlsruhe Institute of Technology, Institute of Organic Chemistry, Fritz-Haber-Weg 6, 76131 Karlsruhe, Germany. *Correspondence: claudia.muhle-goll@kit.edu
}

\section{SUPPORTING INFORMATION}

Table S1: Structure statistics of APP WT and four single point mutants. All values refer to the ensemble of 20 structures with the lowest energy from 400 calculated structures.

\begin{tabular}{|c|c|c|c|c|c|}
\hline & WT & G38L & G38P & V44M & 145T \\
\hline Total restraints used & 416 & 406 & 452 & 378 & 356 \\
\hline unambigous NOE restraints & 371 & 376 & 395 & 352 & 324 \\
\hline Intraresidue & 161 & 168 & 210 & 153 & 134 \\
\hline Sequential $(|i-j|=1)$ & 108 & 100 & 103 & 94 & 97 \\
\hline Medium range $(1<|i-j|<4)$ & 88 & 89 & 66 & 86 & 73 \\
\hline Long range $(|i-j| \geq 4)$ & 14 & 19 & 16 & 19 & 20 \\
\hline Ambiguous NOE restraints & 45 & 30 & 57 & 26 & 27 \\
\hline Backbone dihedral angle restraints & 52 & 52 & 52 & 54 & 50 \\
\hline \multicolumn{6}{|l|}{ Statistics for structure calculations } \\
\hline RMSD of bonds (A) & $0.001+/-0.00007$ & $0.001+/-0.00008$ & $0.001+/-0.00006$ & $0.001+/-0.00015$ & $0.001+/-0.00005$ \\
\hline RMSD of bond angles $\left({ }^{\circ}\right)$ & $0.257+/-0.005$ & $0.290+/-0.009$ & $0.279+/-0.007$ & $0.259+/-0.006$ & $0.260+/-0.003$ \\
\hline RMSD of improper torsions $\left({ }^{\circ}\right)$ & $0.140+/-0.012$ & $0.170+/-0.018$ & $0.174+/-0.013$ & $0.133+/-0.007$ & $0.139+/-0.009$ \\
\hline \multicolumn{6}{|l|}{ Final Energies $\left(\mathrm{kcal} \mathrm{mol}^{-1}\right)$} \\
\hline$E_{\text {total }}$ & $-1001+/-42$ & $-1048+/-28$ & $-1011+/-28$ & $-1041+/-22$ & $-1028+/-32$ \\
\hline Ebonds & $0.448+/-0.064$ & $0.747+/-0.092$ & $0.633+/-0.063$ & $0.546+/-0.158$ & $0.450+/-0.048$ \\
\hline Eangles & $8.85+/-0.35$ & $11.56+/-0.711$ & $10.6+/-0.5$ & $8.98+/-0.247$ & $8.88+/-0.24$ \\
\hline Eimpropers & $0.616+/-0.107$ & $0.936+/-0.200$ & $0.973+/-0.146$ & $0.555+/-0.062$ & $0.598+/-0.074$ \\
\hline Edihed & $121.5+/-1.2$ & $134.5+/-2.4$ & $124.0+/-1.0$ & $122.9+/-2.0$ & $122.5+/-1.50$ \\
\hline 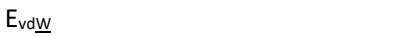 & $-229.6+/-3.2$ & $-232.8+/-2.9$ & $-235.4+/-2.8$ & $-227.8+/-3.5$ & $-226.3+/-3.0$ \\
\hline $\mathrm{E}_{\mathrm{NOE}}$ & $-902.8+/-42.3$ & $-962.9+/-26.8$ & $-912.2+/-28.2$ & $-945.9+/-22.0$ & $-934.4+/-32.5$ \\
\hline \multicolumn{6}{|l|}{ Coordinate precision (A) } \\
\hline $\begin{array}{l}\text { RMSD of backbone }(N, C A, C, O) \text { of all } \\
\text { residues }\end{array}$ & $2.10+/-0.69$ & $1.84+/-0.54$ & $1.76+/-0.62$ & $2.23+/-0.65$ & $2.09+/-0.76$ \\
\hline $\begin{array}{l}\text { RMSD of all heavy atoms of all } \\
\text { residues }\end{array}$ & $2.53+/-0.66$ & $2.16+/-0.56$ & $2.12+/-0.70$ & $2.57+/-0.72$ & $2.40+/-0.74$ \\
\hline $\begin{array}{l}\text { RMSD of backbone }(N, C A, C, O) \text { of } \\
\text { ordered residues }(29: 54)\end{array}$ & $1.43+/-0.48$ & $1.31+/-0.39$ & $1.44+/-0.55$ & $1.77+/-0.59$ & $1.40+/-0.54$ \\
\hline $\begin{array}{l}\text { RMSD of all heavy atoms of } \\
\text { ordered residues (29:54) }\end{array}$ & $1.65+/-0.47$ & $1.53+/-0.39$ & $1.79+/-0.64$ & $2.09+/-0.61$ & $1.61+/-0.47$ \\
\hline
\end{tabular}


Table S2: Kink and swivel angles of 20 lowest energy structures of APP WT and four mutants. Swivel angles in brackets were omitted from mean calculation due to too small kink angles to determine swivel direction correctly.

\begin{tabular}{|c|c|c|c|}
\hline WT & $\operatorname{Kink} \theta\left[^{\circ}\right]$ & Swivel $\varphi\left[^{\circ}\right]$ & \multirow{23}{*}{$\begin{array}{l}\text { Top view of WT } 8 \text { (orange) and WT } 9 \text { (black). } \\
\text { Due to small kink angles, the great difference in } \\
\text { swivel angles }\left(64^{\circ} \text { vs }-79^{\circ} \text { ) is not }\right. \\
\text { distinguishable. Therefore, swivel angles of } \\
\text { structures with small kink were omitted from } \\
\text { mean calculation. }\end{array}$} \\
\hline wt 1 & 61.814 & 87.858 & \\
\hline wt 2 & 58.031 & -3.946 & \\
\hline wt 3 & 19.203 & (65.588) & \\
\hline wt 4 & 71.806 & 46.289 & \\
\hline wt 5 & 51.353 & 36.41 & \\
\hline wt 6 & 13.816 & (42.461) & \\
\hline wt 7 & 45.618 & 32.382 & \\
\hline wt 8 & 11.385 & (64.748) & \\
\hline wt 9 & 5.042 & $(-79.398)$ & \\
\hline wt 10 & 12.395 & (73.477) & \\
\hline wt 11 & 40.113 & -4.711 & \\
\hline wt 12 & 69.788 & 23.553 & \\
\hline wt 13 & 11.368 & $(-96.876)$ & \\
\hline wt 14 & 49.059 & 61.344 & \\
\hline wt 15 & 22.396 & 14.434 & \\
\hline wt 16 & 64.93 & 20.235 & \\
\hline wt 17 & 43.594 & 44.707 & \\
\hline wt 18 & 43.967 & 10.241 & \\
\hline wt 19 & 25.25 & 14.554 & \\
\hline wt 20 & 43.763 & 32.263 & \\
\hline mean & 38.2 & $29.7(24.3)$ & \\
\hline mean deviation & 18.5 & $19.1(32.0)$ & \\
\hline
\end{tabular}

\begin{tabular}{|c|c|c|}
\hline G38L & Kink $\theta\left[{ }^{\circ}\right]$ & Swivel $\varphi\left[^{\circ}\right]$ \\
\hline g38l 1 & 35.673 & -169.179 \\
\hline g381 2 & 3.644 & $(-269.761)$ \\
\hline g381 3 & 39.385 & -11.492 \\
\hline g381 4 & 7.268 & $(-132.663)$ \\
\hline g381 5 & 10.783 & -87.674 \\
\hline g381 6 & 29.704 & -39.214 \\
\hline g381 7 & 14.356 & $(151.472)$ \\
\hline g381 8 & 4.391 & $(-160.097)$ \\
\hline g381 9 & 44.495 & -80.275 \\
\hline g381 10 & 36.72 & -67.394 \\
\hline g38I 11 & 51.609 & -44.572 \\
\hline g38I 12 & 30.561 & -98.709 \\
\hline g38l 13 & 23.937 & -111.34 \\
\hline g38I 14 & 12.987 & -95.304 \\
\hline g38l 15 & 22.476 & $(28.758)$ \\
\hline g38I 16 & 25.187 & (65.715) \\
\hline g38I 17 & 31.531 & -79.548 \\
\hline g38l 18 & 17.373 & $(-23.347)$ \\
\hline g38l 19 & 57.157 & -56.046 \\
\hline g381 20 & 16.892 & $(197.02)$ \\
\hline mean & 25.8 & $-81.4(-54.2)$ \\
\hline mean deviation & 12.5 & $35.3(75.8)$ \\
\hline
\end{tabular}

\begin{tabular}{|l|r|r|}
\hline \multicolumn{1}{|c|}{ G38P } & Kink $\boldsymbol{\theta}\left[^{\circ}{ }^{\text {] }}\right.$ & Swivel $\boldsymbol{\varphi}\left[^{\circ}{ }^{\circ}\right.$ \\
\hline g38p 1 & 17.68 & -7.962 \\
g38p 2 & 44.489 & -100.859 \\
g38p 3 & 16.015 & -121.887 \\
g38p 4 & 41.057 & -73.591 \\
g38p 5 & 8.16 & -125.587 \\
g38p 6 & 25.663 & -123.232 \\
g38p 7 & 53.027 & -53.441 \\
g38p 8 & 43.719 & -87.079 \\
g38p 9 & 3.646 & 96.687 \\
g38p 10 & 84.539 & -101.979 \\
g38p 11 & 32.658 & -68.799 \\
g38p 12 & 35.107 & -64.193 \\
g38p 13 & 34.532 & -57.445 \\
g38p 14 & 61.336 & -66.356 \\
g38p 15 & 47.45 & -62.879 \\
g38p 16 & 23.644 & -64.477 \\
g38p 17 & 18.442 & -252.13 \\
g38p 18 & 25.006 & -38.325 \\
g38p 19 & 33.411 & -102.796 \\
g38p 20 & 63.519 & -122.947 \\
\hline mean & 35.7 & -80.0 \\
mean deviation & 15.4 & 41.9 \\
\hline
\end{tabular}




\begin{tabular}{|l|r|r|}
\hline \multicolumn{1}{|c|}{ V44M } & \multicolumn{1}{c|}{ bend } & \multicolumn{1}{c|}{ swivel } \\
\hline v44m 1 & 31.238 & 5.356 \\
v44m 2 & 52.666 & -42.731 \\
v44m 3 & 25.005 & -159.131 \\
v44m 4 & 46.944 & -5.565 \\
v44m 5 & 49.573 & -97.385 \\
v44m 6 & 45.203 & -88.707 \\
v44m 7 & 31.169 & 160.676 \\
v44m 8 & 15.816 & -28.524 \\
v44m 9 & 32.672 & -45.351 \\
v44m 10 & 14.529 & $(-100.681)$ \\
v44m 11 & 68.901 & -9.369 \\
v44m 12 & 13.399 & $(65.168)$ \\
v44m 13 & 6.474 & $(66.948)$ \\
v44m 14 & 50.929 & 26.13 \\
v44m 15 & 25.676 & 80.297 \\
v44m 16 & 26.924 & -142.789 \\
v44m 17 & 14.444 & -159.07 \\
v44m 18 & 20.775 & 123.573 \\
v44m 19 & 25.999 & 123.602 \\
v44m 20 & 3.737 & -164.275 \\
\hline mean & 30.1 & $-24.9(-3.2)$ \\
mean deviation & 13.8 & $82.8(84.5)$ \\
\hline
\end{tabular}

\begin{tabular}{|l|r|r|}
\hline \multicolumn{1}{|c|}{ I45T } & \multicolumn{1}{c|}{ bend } & \multicolumn{1}{c|}{ swivel } \\
\hline i45t 1 & 41.884 & 92.656 \\
i45t 2 & 30.759 & -116.961 \\
i45t 3 & 50.011 & 151.8 \\
i45t 4 & 17.141 & $(105.942)$ \\
i45t 5 & 36.593 & 138.018 \\
i45t 6 & 32.909 & -166.831 \\
i45t 7 & 21.451 & $(79.205)$ \\
i45t 8 & 39.488 & 19.761 \\
i45t 9 & 41.696 & 99.035 \\
i45t 10 & 33.22 & 93.538 \\
i45t 11 & 9.996 & $(-45.364)$ \\
i45t 12 & 7.56 & $(94.5)$ \\
i45t 13 & 21.862 & 143.071 \\
i45t 14 & 15.125 & -73.219 \\
i45t 15 & 34.27 & 116.612 \\
i45t 16 & 11.228 & $(108.546)$ \\
i45t 17 & 16 & $(120.381)$ \\
i45t 18 & 33.645 & 154.23 \\
i45t 19 & 58.575 & 148.098 \\
i45t 20 & 17.289 & $(48.965)$ \\
\hline mean & 28.5 & $61.5(65.6)$ \\
mean deviation & 11.9 & $89.7(72.7)$ \\
\hline
\end{tabular}


Table S3: All-residue C $\alpha$ RMSDs of the NMR structures with respect to C99 in the theoretical substrateenzyme encounter model. All values given in $\AA$.

\begin{tabular}{|c|c|c|c|c|c|}
\hline Model \# & WT & V44M & G38L & G38P & 145T \\
\hline 1 & 7.25 & 8.33 & 14.55 & 15.18 & 6.92 \\
\hline 2 & 7.82 & 12.29 & 11.26 & 12.35 & 12.41 \\
\hline 3 & 10.46 & 12.59 & 10.09 & 11.87 & 11.46 \\
\hline 4 & 4.00 & 8.17 & 10.64 & 10.27 & 9.06 \\
\hline 5 & 6.43 & 15.15 & 12.37 & 13.87 & 10.55 \\
\hline 6 & 10.36 & 13.46 & 11.95 & 12.11 & 12.77 \\
\hline 7 & 6.11 & 11.60 & 9.62 & 12.76 & 8.51 \\
\hline 8 & 10.01 & 10.65 & 10.54 & 13.12 & 7.00 \\
\hline 9 & 11.78 & 11.10 & 10.05 & 13.57 & 9.07 \\
\hline 10 & 10.30 & 10.81 & 12.36 & 12.24 & 6.72 \\
\hline 11 & 8.59 & 8.93 & 11.96 & 11.42 & 10.88 \\
\hline 12 & 4.43 & 8.49 & 13.84 & 13.56 & 10.31 \\
\hline 13 & 13.11 & 9.67 & 13.44 & 12.75 & 11.12 \\
\hline 14 & 6.24 & 5.56 & 12.73 & 11.00 & 10.85 \\
\hline 15 & 8.94 & 8.19 & 9.35 & 14.68 & 9.87 \\
\hline 16 & 5.80 & 14.22 & 9.19 & 12.67 & 11.24 \\
\hline 17 & 5.76 & 12.06 & 12.63 & 16.50 & 10.16 \\
\hline 18 & 7.40 & 10.11 & 9.70 & 15.08 & 10.22 \\
\hline 19 & 9.69 & 10.02 & 13.25 & 10.86 & 12.82 \\
\hline 20 & 7.02 & 11.29 & 13.23 & 11.76 & 8.15 \\
\hline Average & 8.07 & 10.63 & 11.64 & 12.88 & 10.00 \\
\hline Std. Dev. & 2.40 & 2.28 & 1.60 & 1.56 & 1.80 \\
\hline
\end{tabular}


Figure S1. Secondary chemical shifts. A) ${ }^{1} \mathrm{H} \alpha$ chemical shifts of all five peptides relative to random coil chemical shifts. To highlight changes in helicity, differences between ${ }^{1} \mathrm{H} \alpha$ secondary chemical shifts of each mutant and the wild type were calculated. B) Same for ${ }^{13} \mathrm{C} \alpha$ and C) for ${ }^{13} \mathrm{C} \beta$ secondary chemical shifts.

A

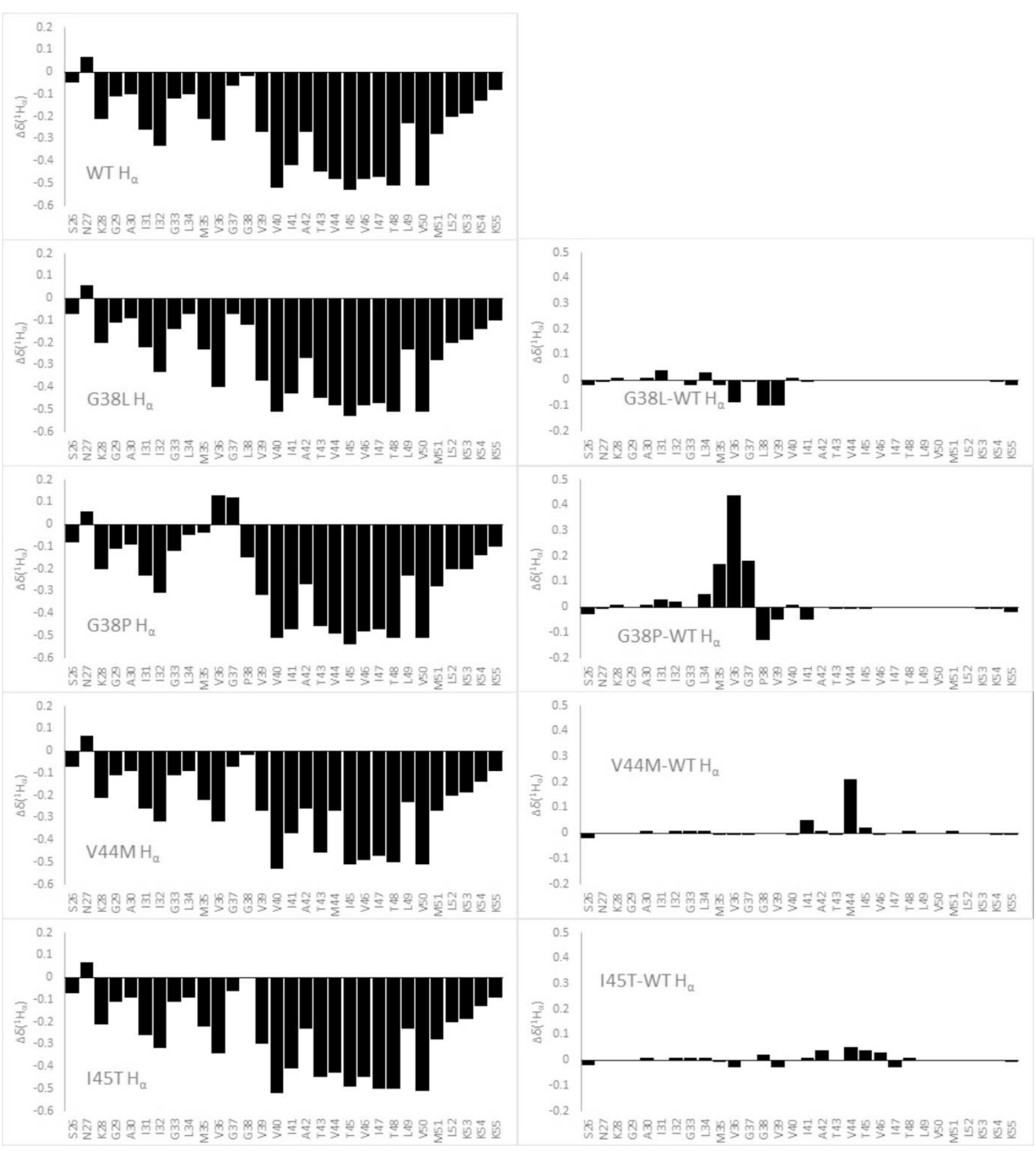




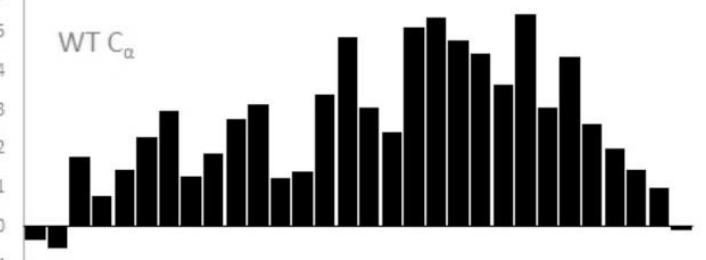

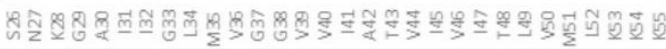

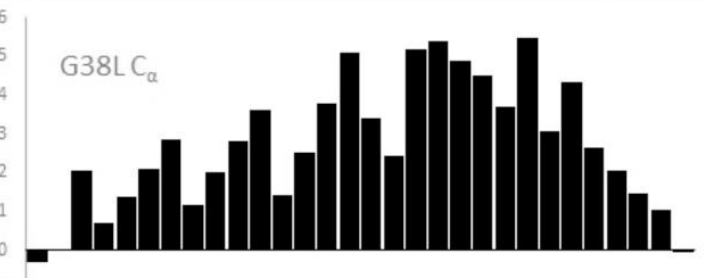

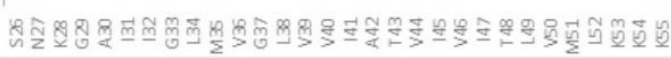

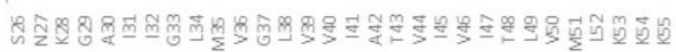

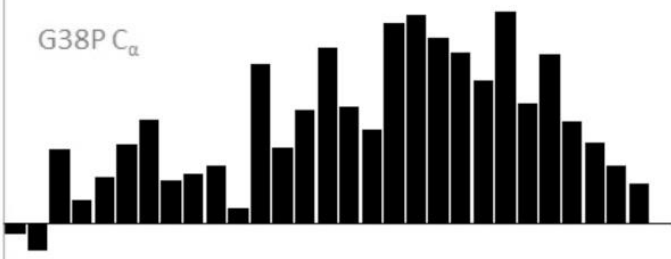

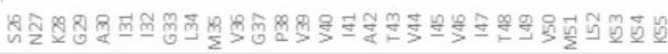

G38L-WT C
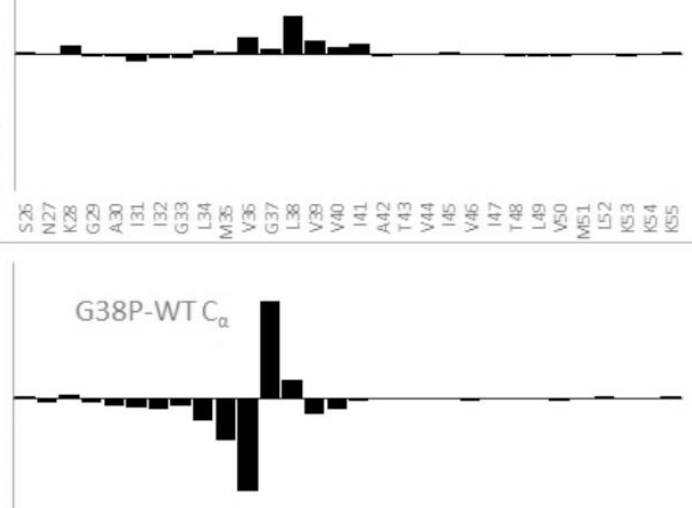

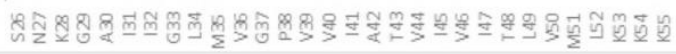

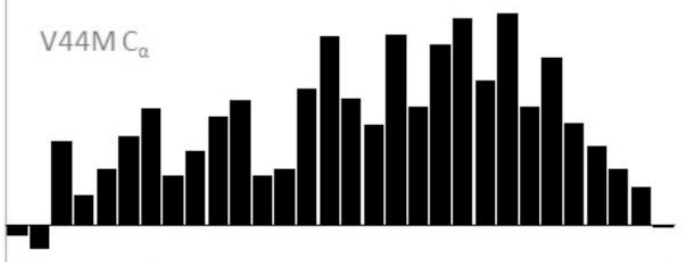

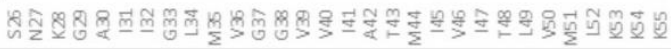

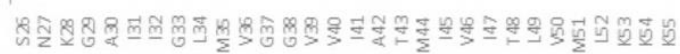

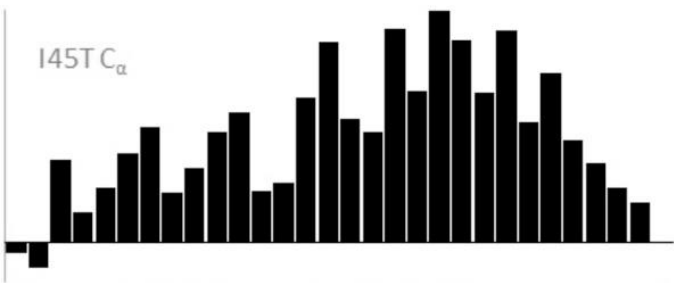

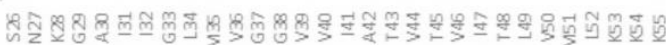

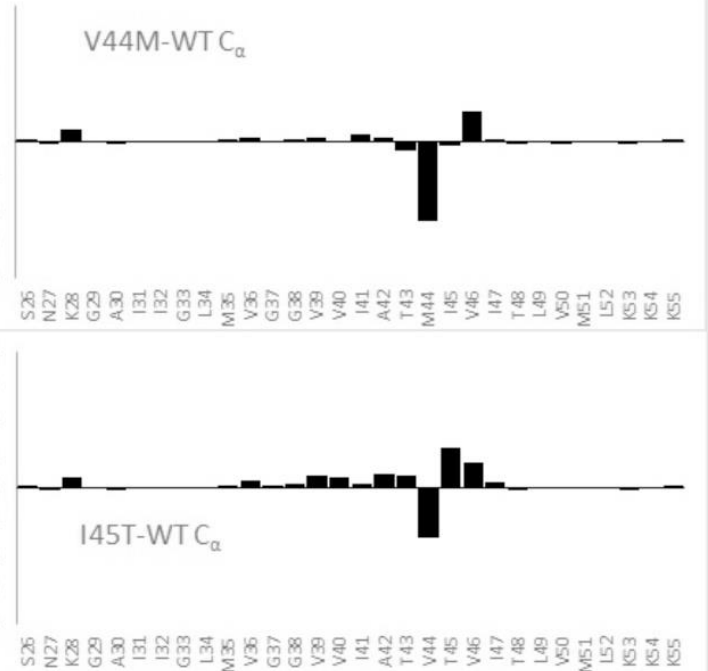


C
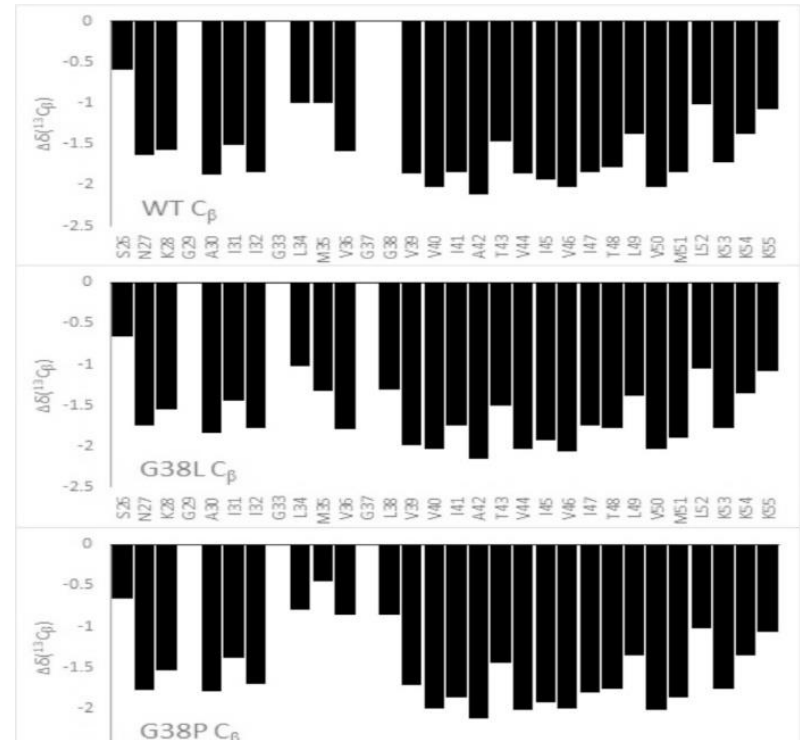

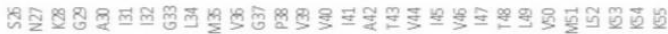
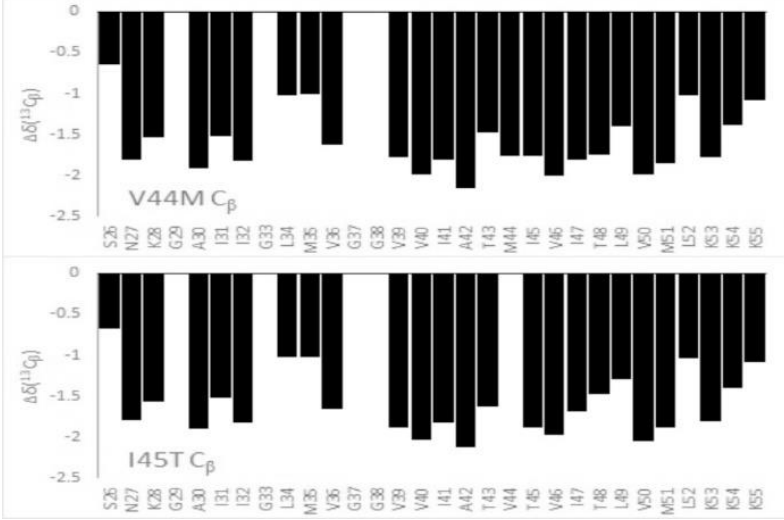
Figure S2. Order parameters $S^{2}$ (left column) and helix probability (right column) for all residues of all five peptides. $S^{2}$ order parameters were predicted from backbone chemical shifts by TALOS $+^{1,2}$. Secondary structure prediction was derived from TALOS+ based on an artificial neural network.

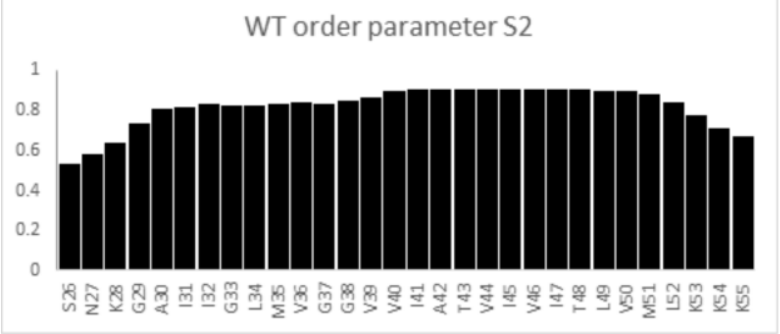

G38L order parameter S2

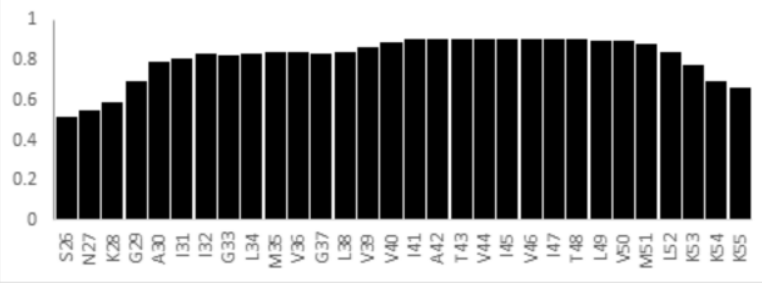

G38P order parameter S2

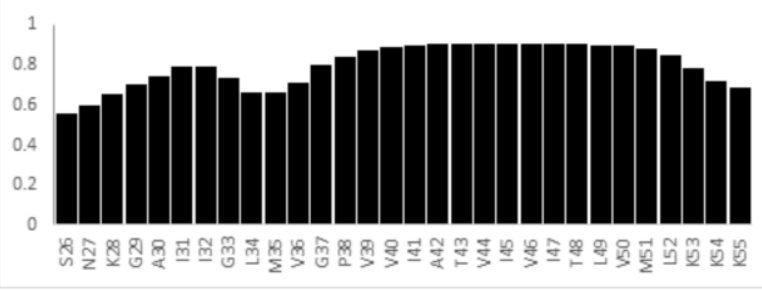

V44M order parameter S2

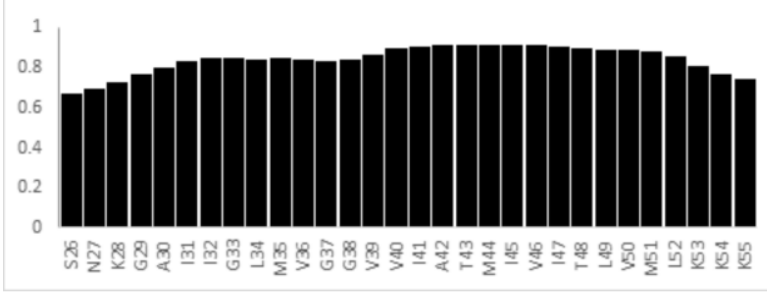

145T order parameter S2

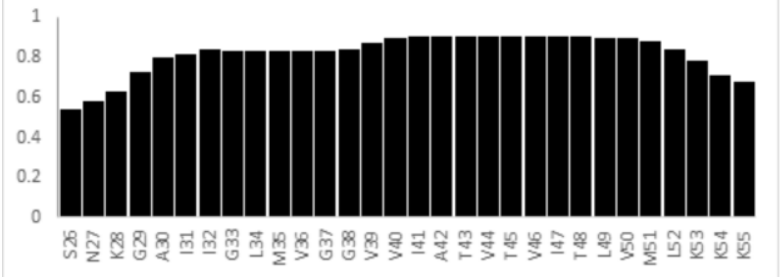

WT helix probability

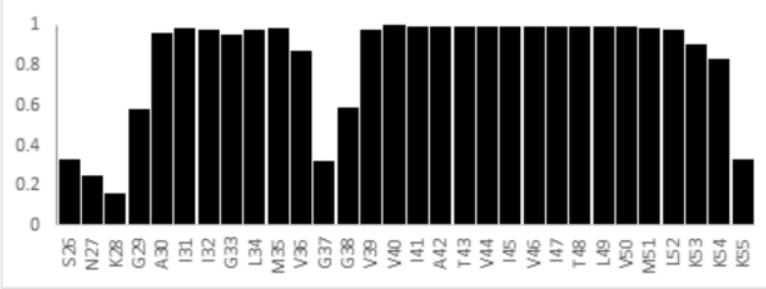

G38L helix probability

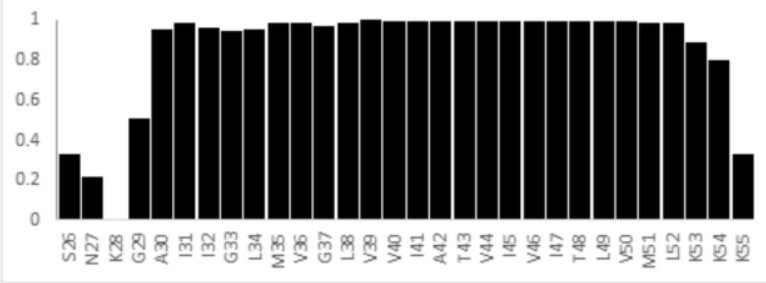

G38P helix probability

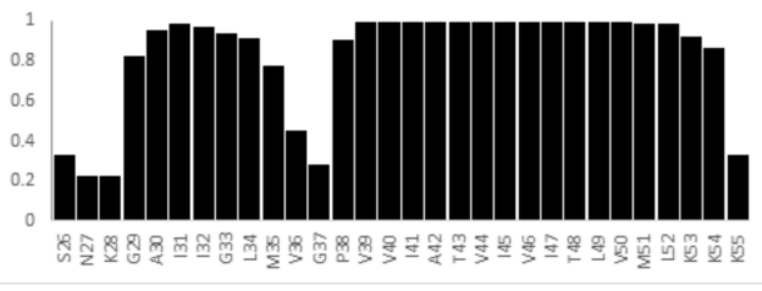

V44M helix probability

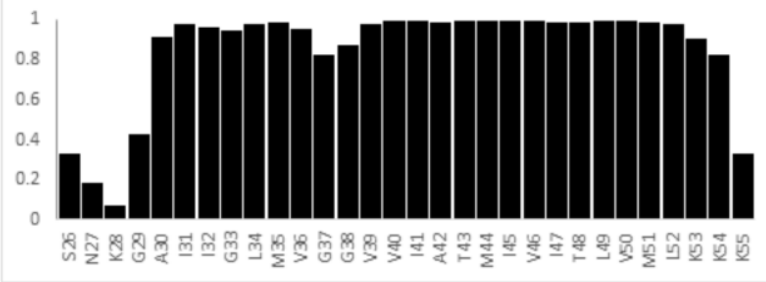

I45T helix probability

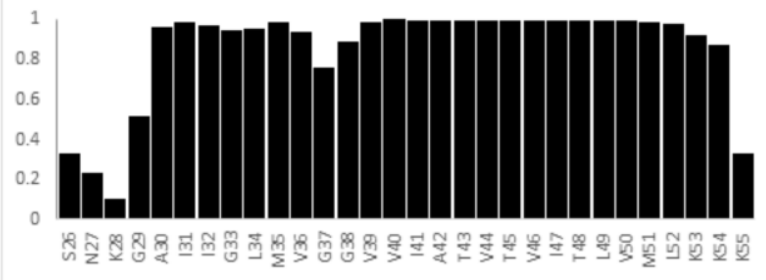


Figure S3. Distribution of water molecules in the cryo-EM structure 6IYC and the MD model. Both snapshots are actually taken from MD simulations. Simulation details of the MD model have already been published elsewhere ${ }^{3}$.

The snapshot of the 6IYC structure is the last frame of a short 40 ns MD simulation. The starting structure of this simulation has been generated using the lipid builder of the CHARMM-GUI webserver ${ }^{4}$. The solvent consisted of 304 POPC molecules (membrane) and 60309 water molecules as well as $0.15 \mathrm{M} \mathrm{KCl}$. For consistency, we used the same force fields, as well as the same simulation settings as in the case of the already published MD substrate-encounter model ${ }^{3}$.

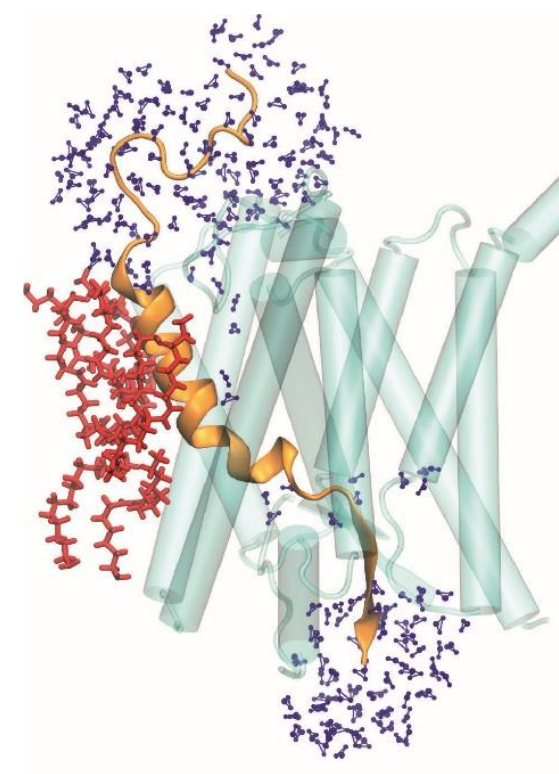

6IYC

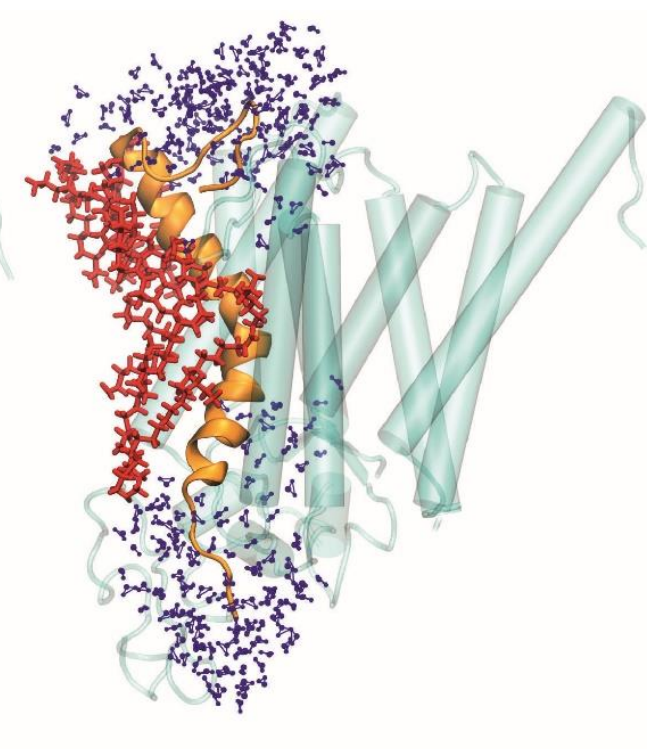

MD model 
Figure S4. Schematic model of $\gamma$-secretase substrate interactions that result in first cleavage at the $\varepsilon$ site. 1. Initial substrate encounter complex. Here the substrate TM helix is still straight as observed in lipid bilayers and detergent micelles. 2. Threading of substrate into enzyme cavity. To achieve this the TM helix needs to bend. Correct mutual orientation of the two helical segments facilitates threading, 3. Positioning at the active site and subsequent $\varepsilon$-cleavage. Presenilin (PS1) TM helices outlining the binding site are colored in dark blue while other PS1 helices, not interacting with C99 are depicted in light blue. PEN2 is given in green, APP-TMD in orange. The catalytic aspartates are depicted in red.
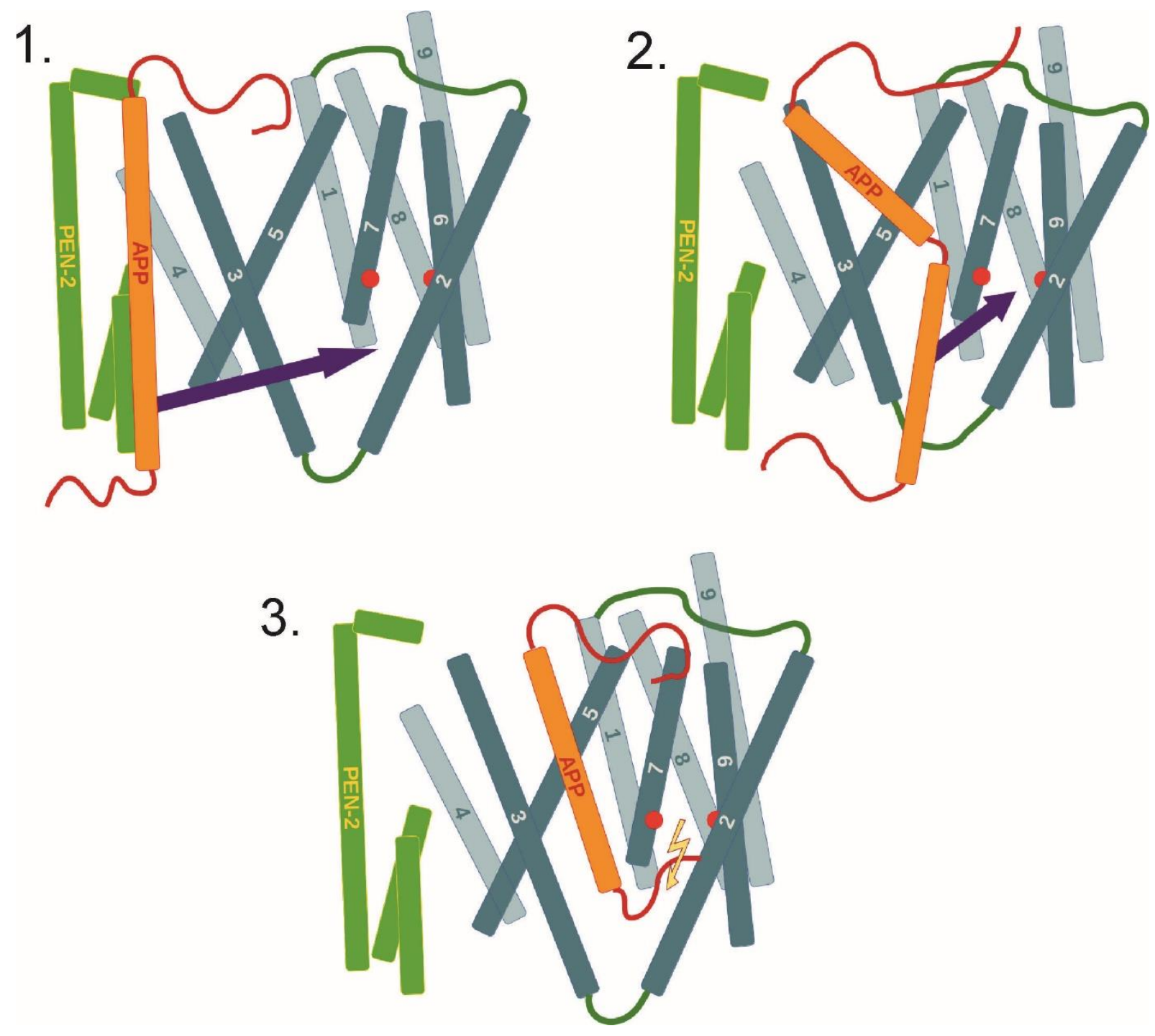
Figure S5. Alignment of the NMR structures with substrate-bound APP-TM of the cryo-EM structure 6IYC.

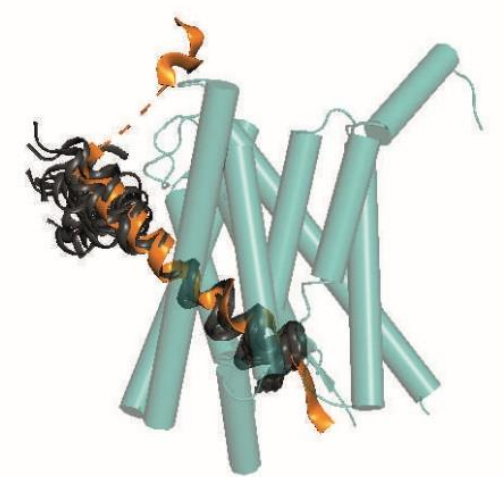

WT

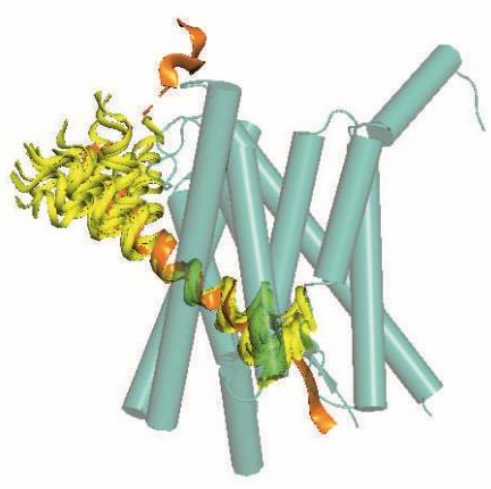

V44M

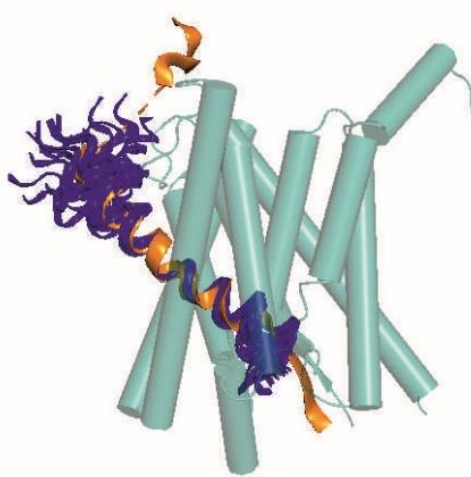

G38L

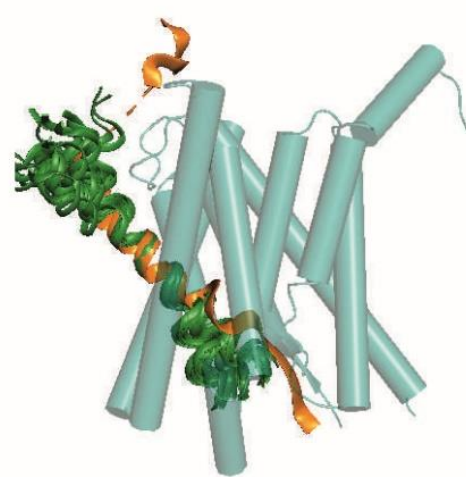

145T

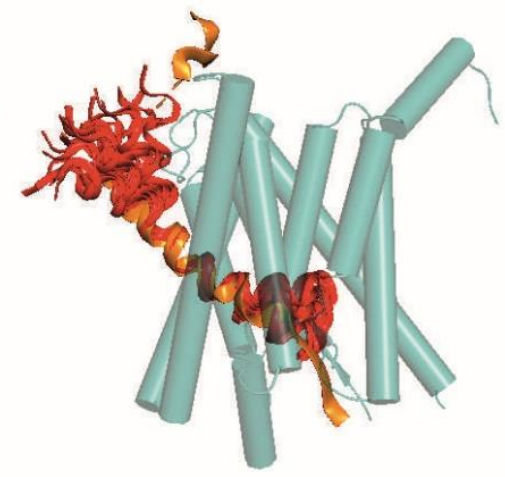

G38P

\section{REFERENCES}

1. Shen, Y., Delaglio, F., Cornilescu, G. \& Bax, A. TALOS+: a hybrid method for predicting protein backbone torsion angles from NMR chemical shifts. J. Biomol. NMR 44, 213-223, doi:10.1007/s10858009-9333-z (2009).

2. Berjanskii MV and Wishart DS A simple method to predict protein flexibility using secondary chemical shifts. J. Am. Chem. Soc. 127, 14970-14971. doi: 10.1021/ja054842f (2005).

3. Hitzenberger, M. \& Zacharias, M. Structural Modeling of gamma-Secretase Abeta $n$ Complex Formation and Substrate Processing. ACS Chem Neurosci 10, 1826-1840, doi:10.1021/acschemneuro.8b00725 (2019).

4. Lee, J. et al. CHARMM-GUI Membrane Builder for Complex Biological Membrane Simulations with Glycolipids and Lipoglycans. J. Chem. Theory Comput. 15, 775-786 doi:10.1021/acs.jctc.8b01066 (2019). 